\title{
Optical coherence tomography in multiple sclerosis
}

\author{
Josefine Britze ${ }^{1}$. Jette Lautrup Frederiksen ${ }^{1}$
}

Received: 10 November 2017 / Accepted: 15 November 2017 / Published online: 2 February 2018

(c) The Royal College of Ophthalmologists 2018

\begin{abstract}
To summarize recent findings regarding the utility of optical coherence tomography in multiple sclerosis. We searched PubMed for relevant articles using the keywords 'optical coherence tomography multiple sclerosis'. Additional articles were found via references in these articles. We selected articles based on relevance. Optical coherence tomography has contributed to greater insights into the pathophysiology of multiple sclerosis. Loss of retinal nerve fibre layer and ganglion cell layer thickness correlate with clinical and paraclinical parameters such as visual function, disability and magnetic resonance imaging. Some studies indicate that OCT parameters may be able to predict disability progression and visual function in MS. OCT angiography has recently emerged as a novel technique to study MS. OCT has proven very useful with regards to research, monitoring and predicting disability in multiple sclerosis. It will be interesting to see how OCT angiography will contribute to this field.
\end{abstract}

\section{Introduction}

Multiple sclerosis (MS) is characterized by both inflammation and axonal degeneration. Visual symptoms are common and optic neuritis $(\mathrm{ON})$ is the onset symptom in $\sim 20 \%$ of patients with MS. Even patients who have not experienced ON, will most likely also have lesions in their visual pathways, as post-mortem studies show that this is the case for $90 \%$ of patients regardless of ON history $[1,2]$.

The anterior visual pathways consist of the retinal ganglion cells, whose somas are in the ganglion cell layer (GCL). Their axons form the retinal nerve fibre layer (RNFL) and are unmyelinated until they leave the eye. These axons form the optic nerve, which travels via the optic canal to the optic chiasm where the nasal fibres decussate and most of the fibres then go on to synapse at the lateral geniculate nucleus.

The GCL and RNFL are very small, but can be imaged using optical coherence tomography (OCT), which is a

Jette Lautrup Frederiksen

jette.lautrup.frederiksen.battistini@regionh.dk

1 Department of Neurology, Rigshospitalet Glostrup, Faculty of Health and Medical Sciences, University of Copenhagen, Glostrup, Denmark technique that uses near-infrared light to create images of the retina. It is non-invasive, quick, and relatively cheap and easy to use. Furthermore, the images produced via OCT are of very high resolution and highly reproducible. Assessment of the RNFL and GCL using OCT potentially allows us to assess axonal and neuronal degeneration. The technique has therefore been investigated in relation to MS. Many studies have shown that both the RNFL and the GCL are statistically significantly reduced in patients with MS, both with (MSON) and without (MS-NON) prior ON $[3,4]$. In this article, we review the ways in which this technique has proven useful in studying and treating MS patients.

\section{Optical coherence tomography}

The first OCT devices used time domain technology, which could measure the thickness of the RNFL. As such, this layer was the focus of most early research. However, with the advent of spectral domain OCT technology, came the ability to assess other retinal layers, such as the ganglion cell layer. Most current segmentation algorithms cannot accurately measure the thickness of the GCL by itself, but instead measure the thickness of the composite GCL and inner plexiform layer (GCIPL). Other retinal layers such as the photoreceptor layer and the outer nuclear layer have also been subjects of research lately. 


\section{Pathophysiological insights}

OCT has been used to obtain more knowledge about the pathophysiological mechanisms involved in MS. OCT can be used to image the neuronal and unmyelinated axonal layers of the retina. As such, it provides an easy-to-use model to study neurodegeneration in MS. The high degree of reproducibility and non-invasive character makes OCT especially well-suited for research purposes.

The pathological mechanism behind the reduction in RNFL and GCIPL thickness in MS patients both with and without prior $\mathrm{ON}$ is still in dispute. In patients with prior $\mathrm{ON}$, the most likely explanation is loss of axons due to retrograde degeneration after ON. However, in MS patients who have not had clinical ON, and who typically display a RNFL and GCIPL loss of $\sim 6.73 \mu \mathrm{m}$ [4], several different mechanisms have been proposed. Some suggest the loss of GCIPL and RNFL thickness is caused by retrograde degeneration after a mild, subclinical ON. Others have proposed that it could be caused by primary degeneration of the GCIPL neurons due to MS. Lastly, many have suggested that lesions in the optic radiation via trans-synaptic degeneration could lead to loss of retinal ganglion cells and their axons. Indeed, Puthenparampil et al. [5] found an inverse correlation between the thickness of the temporal RNFL and the ipsilateral optic radiation white matter lesion load $(r=-0.7, p<0.05)$ in patients with MSON. Furthermore, they observed an inverse correlation between the nasal RNFL and the contralateral optic radiation white matter lesion load $(r=-0.8, p<0.01$ for both the superior and inferior nasal quadrant). OCT angiography, although still in the developing phase, may facilitate studies of neurovascular coupling in MS.

\section{Diagnostic relevance}

Changes in the GCIPL and the RNFL are commonly thought to reflect oedema and neurodegeneration. These phenomena are not specific for MS and as such it is important that such findings are interpreted contextually. However, many studies indicate that the temporal development, severity and distribution of changes in the GCIPL and RNFL may help differentiate between MS and some differential diagnoses. In neuromyelitis optica spectrum disorder, the reduction in GCIPL and RNFL thickness is usually much more severe than in MSON [6]. Furthermore, the distribution of RNFL loss tends to involve the temporal quadrant in MSON, whereas it is more diffusely distributed in neuromyelitis optica spectrum disorder [6]. In Susac syndrome, the pattern of GCIPL and RNFL loss may help differentiate it from MS [7, 8]. These observations can be found in patients long after their branch retinal arterial occlusion, which makes OCT more useful than fluorescein angiography, which will only detect a branch retinal arterial occlusion in the acute phase [8]. In idiopathic intracranial hypertension, there is also swelling of the optic nerve head. However, unlike in MS, this is usually symmetrical [9].

Many studies have found statistically significant reductions in the GCL thickness in eyes of MS patients without prior ON, which may reflect subclinical structural damage. Some have suggested that this could help identify patients with ON who are at risk of developing MS. As such, Coric et al. [10] investigated whether the inter-eye percentage difference (IEPD) could be useful in distinguishing MS patients from healthy controls. They found that the diagnostic accuracy of the IEPD (area under the curve) was $0.73-0.86$ for the RNFL and $0.75-0.94$ for the GCIPL. The diagnostic sensitivity of the GCIPL IEPD was $70 \%$ with a specificity of $97 \%$ for distinguishing unilateral MSON from healthy controls. The IEPD of the GCIPL could distinguish healthy controls and MS-NON. The IEDP has potential to be a very useful parameter clinically, as it is dimensionless and therefore could overcome problems with using different devices and segmentation algorithms. It could also overcome intrapersonal factors such as ethnicity and physiological variability. This study also found that the IEPD of the GCIPL was superior to that of the RNFL, which the authors suggest reflects more variability within the RNFL. However, the clinical usefulness of this is unclear.

\section{Monitoring MS}

Many studies have found correlations between OCT parameters and clinical and paraclinical aspects in MS. This suggests that OCT could be a useful tool for monitoring MS both in clinic and in treatment trials.

\section{Biomarkers}

Knier et al. observed an association between low GCIPL volumes and increased intrathecal B-cell frequencies and intrathecal IgG synthesis [11].

\section{Disease subtype}

OCT could potentially help differentiate between MS subtypes. One multicentre study [12] looked at 571 eyes of MS patients without prior ON and they observed a lower RNFL thickness in patients with secondary progressive MS (SPMS) compared to relapsing-remitting MS (RRMS) $(p=$ 0.007). Furthermore, total macular volume was reduced in SPMS and primary progressive MS (PPMS) eyes compared to RRMS eyes (SPMS: $p=0.039$, PPMS: $p=0.005$ ). There was no statistically significant difference between the RNFL 
thickness and total macular volume in patients with SPMS and PPMS. When the authors corrected for expanded disability status scale (EDSS) score in generalized estimation equation models, these differences disappeared. Similarly, Costello et al. [13] found significantly lower RNFL thicknesses in eyes unaffected by prior ON, in patients with SPMS than with RRMS, and with RRMS than with clinically isolated syndrome (CIS). A similar trend could be detected in eyes with prior ON. In a group of MS patients with and without prior ON, Saidha et al. [14] observed lower GCIPL and RNFL values in SPMS compared to RRMS. However, after correcting for disease duration this was only significant with regards to the GCIPL $(p=0.04)$.

\section{Visual function}

Many studies have found correlations between the thickness of the GCIPL and the RNFL, and visual acuity, especially low contrast visual acuity [14-16]. One study also detected a moderate, statistically significant correlation between dyschromatopsia and GCIPL thickness in MS-NON eyes [17].

\section{Clinical disability}

Some studies have found significant inverse correlations between GCIPL thickness and EDSS [14, 18-22], whereas others have not [23-25]. One study [26] also found an association between increased rate of GCIPL thinning and EDSS score progression, which others have failed to replicate [27].

\section{Magnetic resonance imaging (MRI)}

MRI studies have found several associations between RNFL thickness and MRI parameters in MS patients [2730]. Furthermore, recent studies have observed correlations between GCIPL thickness and thalamus volume [31], normalized brain parenchymal volume [17], intracranial volume [22], cortical grey matter volume [22], and number and volume of cortical lesions [31]. Additionally, studies have found associations between increased thinning of the GCIPL and T2 lesions [26], increased lesion volume in the optic radiation [32] and development of contrast-enhancing lesions [26, 27]. The rate of GCIPL thinning has been associated with atrophy of the white matter, cortical grey matter, thalamic, brainstem, and the whole brain [27]. In MS-NON eyes only, studies have found correlations between GCIPL thickness and normalized grey matter volume [32, 33], caudate volume [22], normalized brain volume [33], fractional anisotropy [32], and normalized white matter volume [32,33]. One recent study found an association between thinning of the RNFL and new lesions in the optic radiation [34].

\section{Neurophysiology}

One study found a significant correlation between GCIPL thickness and inverse correlation with multifocal VEP latency ( $r=-0.48, p=0.0001)$ in the eyes of MS patients without prior ON [35]. Similarly, Behbehani et al. [36] observed significant inverse correlations between the average RNFL and GCL thickness and full field VEP latency in RRMS patients.

\section{Temporal evolution}

Several studies have found a statistically significant inverse correlation between disease duration and GCIPL thickness $[14,18,26]$. Others have found correlations between disease duration and the rate of GCIPL thinning in RRMS [21] and non-ON RRMS only [37].

\section{Pupillary response}

One study looked at the pupillary light reflex in MS patients and found that attenuation of the melanopsin-mediated sustained pupillary constriction response was significantly associated with thinning of the GCIPL part of the retina, especially in patients with a history of acute ON [38].

\section{Prognostic value}

During recent years, a greater number of studies have indicated that OCT parameters may be used to predict future visual function and disability worsening in MS and CIS patients.

\section{Disability worsening}

Martinez-Lapiscina et al. [39] observed that a baseline RNFL thickness in MS-NON eyes of $\leq 87 \mu \mathrm{m}$ (Cirrus) or 88 $\mu \mathrm{m}$ (Spectralis) approximately doubled the risk of disability worsening during the $2 \mathrm{nd}$ and $3 \mathrm{rd}$ years. This increased by four times after 4-5 years of follow-up. Bsteh et al. [40] observed that having a RNFL thickness below $88 \mu \mathrm{m}$ was independently associated with a threefold increased risk of EDSS progression $(p<0.001)$ and a 2.7 -fold increased risk of cognitive decline $(p<0.001)$ within the following 3 years. A different study observed that MS patients with low GCIPL volumes had a 6.4-fold increased risk of disability worsening within the following three years [11].

\section{Visual function}

Several studies have found a correlation between OCT measurements and visual function after acute $\mathrm{ON}$ in relation to MS and CIS. Sanchez-Dalmau et al. found that the change 
in GCIPL and RNFL volume during the first month of acute ON, could explain $47 \%$ of the change in $2.5 \%$ LCVA and $67 \%$ of the change in colour vision acuity after six months. Similarly, Gabilondo et al. [41] found that a reduction in the GCIPL thickness of $\geq 4.5 \mu \mathrm{m}$ from baseline to 1 month, predicted poor $2.5 \% \mathrm{LCVA}$ and $1.25 \% \mathrm{LCVA}$ recovery after 6 months, with a high sensitivity and specificity. Also, a decrease in the GCIPL thickness of $\geq 7 \mu \mathrm{m}$ from baseline to one month, predicted poor visual field and colour vision acuity recovery after six months. Kupersmith et al. [42] found a correlation between the amount of GCIPL thinning during the initial two months of acute $\mathrm{ON}$, and the perimetry mean deviation after 6 months $(r=0.38, i=0.04)$.

\section{OCT angiography}

OCT angiography allows imaging of the vasculature in the retina and the blood flow to the capillary level. This method is completely non-invasive, unlike conventional angiography. Presently, only a few studies have been published that investigate the usefulness of this device in relation to MS. One study has looked at changes in the vessel density in the retina of patients with MS. They found that the vessel density was decreased in MS patients compared to controls and that the vessel density correlated with the EDSS score [43]. A different study [44] used OCT angiography to measure the optic nerve flow index, which can be used to measure the flow velocity and density of the vessels in the capillary networks. It can also measure the area of the large retinal vessels. They found that the optic nerve flow index was reduced in MS patients with and without prior ON, relative to healthy controls ( $p=0.001$ and $p=0.031$, respectively). The reduction in perfusion across the $\mathrm{ONH}$ in patients with MS was especially pronounced in those with prior ON. They did not find any correlation between optic nerve flow index and structural parameters such as RNFL and the ganglion cell complex. The authors suggested that this indicates, that the optic nerve flow index and the RNFL reflect different aspects of retinal MS pathology. However, the clinical significance of this is unclear. Furthermore, the study results are limited by the fact that they discarded a significant portion of their scans due to lack of quality. Last, OCT angiography can be used to identify differential diagnoses. However, as this technique has only recently been applied in an MS setting, further studies are needed to elucidate its usefulness both in MS research and clinical practice.

\section{Conclusion}

Optical coherence tomography has contributed to greater insights into the pathophysiology of MS. Loss of RNFL and
GCIPL thickness correlates with clinical and paraclinical parameters such as biomarkers, visual function, disability and magnetic resonance imaging in MS. Some studies indicate that OCT parameters may be able to predict disability progression and visual function in MS. As such, this technique provides a quick, easy and relatively cheap way to monitor and predict disease progression in MS. It will be interesting to see how OCT angiography will contribute to this field.

\section{Compliance with ethical standards}

Conflict of interest The authors declare that they have no competing interests.

\section{References}

1. Ikuta F, Zimmerman HM. Distribution of plaques in seventy autopsy cases of multiple sclerosis in the United States. Neurology. 1976;26(6 PT2):26-8.

2. Toussaint D, Périer O, Verstappen A, Bervoets S. Clinicopathological study of the visual pathways, eyes, and cerebral hemispheres in 32 cases of disseminated sclerosis. J Clin Neuroophthalmol. 1983;3:211-20.

3. Petzold A, Balcer LJ, Calabresi PA, Costello F, Frohman TC, Frohman EM, et al. Retinal layer segmentation in multiple sclerosis: a systematic review and meta-analysis. Lancet Neurol. 2017; 16:797-812.

4. Britze J, Pihl-Jensen G, Frederiksen JL. Retinal ganglion cell analysis in multiple sclerosis and optic neuritis: a systematic review and meta-analysis. J Neurol. 2017;264:1837-1853.

5. Puthenparampil M, Federle L, Poggiali D, Miante S, Signori A, Pilotto E, et al. Trans-synaptic degeneration in the optic pathway. A study in clinically isolated syndrome and early relapsingremitting multiple sclerosis with or without optic neuritis. Tzekov R, editor. PLoS One. 2017;12:e183957.

6. Schneider E, Zimmermann H, Oberwahrenbrock T, Kaufhold F, Kadas EM, Petzold A, et al. Optical coherence tomography reveals distinct patterns of retinal damage in neuromyelitis optica and multiple sclerosis. PLoS One. 2013;8:e66151.

7. Brandt AU, Zimmermann H, Kaufhold F, Promesberger J, Schippling S, Finis D, et al. Patterns of retinal damage facilitate differential diagnosis between Susac Syndrome and MS. PLoS One. 2012;7:e38741.

8. Ringelstein M, Albrecht P, Kleffner I, Bühn B, Harmel J, Müller A-K, et al. Retinal pathology in Susac syndrome detected by spectral-domain optical coherence tomography. Neurology. 2015;85:610-8.

9. Smith SV, Friedman DI. The idiopathic intracranial hypertension treatment trial: a review of the outcomes. Headache. 2017;57:1303-10.

10. Coric D, Balk LJ, Uitdehaag BMJ, Petzold A. Diagnostic accuracy of optical coherence tomography inter-eye percentage difference for optic neuritis in multiple sclerosis. Eur $\mathbf{J}$ Neurol. 2017;24:1479-1484.

11. Knier B, Leppenetier G, Wetzlmair C, Aly L, Hoshi M-M, Pernpeintner $\mathrm{V}$, et al. Association of retinal architecture, intrathecal immunity, and clinical course in multiple sclerosis. JAMA Neurol. 2017;74:847.

12. Oberwahrenbrock T, Schippling S, Ringelstein M, Kaufhold F, Zimmermann $\mathrm{H}$, Keser $\mathrm{N}$, et al. Retinal damage in multiple 
sclerosis disease subtypes measured by high-resolution optical coherence tomography. Mult Scler Int. 2012;2012:1-10.

13. Costello F, Hodge W, Pan YI, Freedman M, DeMeulemeester C. Differences in retinal nerve fiber layer atrophy between multiple sclerosis subtypes. J Neurol Sci. 2009;281:74-9.

14. Saidha S, Syc SB, Durbin MK, Eckstein C, Oakley JD, Meyer SA, et al. Visual dysfunction in multiple sclerosis correlates better with optical coherence tomography derived estimates of macular ganglion cell layer thickness than peripapillary retinal nerve fiber layer thickness. Mult Scler. 2011;17:1449-63.

15. Sotirchos ES, Seigo MA, Calabresi PA, Saidha S. Comparison of point estimates and average thicknesses of retinal layers measured using manual optical coherence tomography segmentation for quantification of retinal neurodegeneration in multiple sclerosis. Curr Eye Res. 2013;38:224-8.

16. Walter SD, Ishikawa H, Galetta KM, Sakai RE, Feller DJ, Henderson SB, et al. Ganglion cell loss in relation to visual disability in multiple sclerosis. Ophthalmology. 2012;119:1250-7.

17. Lampert EJ, Andorra M, Torres-Torres R, Ortiz-Pérez S, Llufriu $\mathrm{S}$, Sepúlveda $\mathrm{M}$, et al. Color vision impairment in multiple sclerosis points to retinal ganglion cell damage. J Neurol. 2015;262:2491-7.

18. González-López JJ, Rebolleda G, Leal M, Oblanca N, MuñozNegrete FJ, Costa-Frossard L, et al. Comparative diagnostic accuracy of ganglion cell-inner plexiform and retinal nerve fiber layer thickness measures by Cirrus and Spectralis optical coherence tomography in relapsing-remitting multiple sclerosis. Biomed Res Int. 2014;2014:128517.

19. Garcia-Martin E, Polo V, Larrosa JM, Marques ML, Herrero R, Martin J, et al. Retinal layer segmentation in patients with multiple sclerosis using spectral domain optical coherence tomography. Ophthalmology. 2014;121:573-9.

20. Outteryck O, Majed B, Defoort-Dhellemmes S, Vermersch P, Zéphir H. A comparative optical coherence tomography study in neuromyelitis optica spectrum disorder and multiple sclerosis. Mult Scler. 2015;21:1781-93.

21. Tátrai E, Simó M, Iljicsov A, Németh J, Debuc DC, Somfai GM. In vivo evaluation of retinal neurodegeneration in patients with multiple sclerosis. PLoS One. 2012;7:e30922.

22. Saidha S, Sotirchos ES, Oh J, Syc SB, Seigo MA, Shiee N, et al. Relationships between retinal axonal and neuronal measures and global central nervous system pathology in multiple sclerosis. JAMA Neurol. 2013;70:34-43.

23. Esen E, Sizmaz S, Balal M, Yar K, Demirkiran M, Unal I, et al. Evaluation of the Innermost Retinal Layers and Visual Evoked Potentials in Patients with Multiple Sclerosis. Curr Eye Res. 2016;41:1-6.

24. Álvarez-Cermeño JC, Muñoz-Negrete FJ, Costa-Frossard L, de la Maza SS, Villar LM, Rebolleda G. Intrathecal lipid-specific oligoclonal IgM synthesis associates with retinal axonal loss in multiple sclerosis. J Neurol Sci. 2016;360:41-4.

25. Albrecht P, Ringelstein M, Müller AK, Keser N, Dietlein T, Lappas A, et al. Degeneration of retinal layers in multiple sclerosis subtypes quantified by optical coherence tomography. Mult Scler. 2012;18:1422-9.

26. Ratchford JN, Saidha S, Sotirchos ES, Oh JA, Seigo MA, Eckstein $\mathrm{C}$, et al. Active MS is associated with accelerated retinal ganglion cell/inner plexiform layer thinning. Neurology. 2013;80:47-54.

27. Saidha S, Al-Louzi O, Ratchford JN, Bhargava P, Oh J, Newsome $\mathrm{SD}$, et al. Optical coherence tomography reflects brain atrophy in multiple sclerosis: a four-year study. Ann Neurol. 2015;78: 801-13.
28. Gordon-Lipkin E, Chodkowski B, Reich DS, Smith SA, Pulicken $\mathrm{M}$, Balcer LJ, et al. Retinal nerve fiber layer is associated with brain atrophy in multiple sclerosis. Neurology. 2007;69:1603-9.

29. Dörr J, Wernecke KD, Bock M, Gaede G, Wuerfel JT, Pfueller $\mathrm{CF}$, et al. Association of retinal and macular damage with brain atrophy in multiple sclerosis. PLoS One. 2011;6:e18132.

30. Cilingir V, Batur M, Bulut MD, Milanlioglu A, Yılgor A, Batur A, et al. The association between retinal nerve fibre layer thickness and corpus callosum index in different clinical subtypes of multiple sclerosis. Neurol Sci. 2017;38:1223-32.

31. Petracca M, Cordano C, Cellerino M, Button J, Krieger S, Vancea $\mathrm{R}$, et al. Retinal degeneration in primary-progressive multiple sclerosis: A role for cortical lesions? Mult Scler. 2017;23:43-50

32. Balk LJ, Steenwijk MD, Tewarie P, Daams M, Killestein J, Wattjes MP, et al. Bidirectional trans-synaptic axonal degeneration in the visual pathway in multiple sclerosis. J Neurol Neurosurg Psychiatry. 2015;86:419-24.

33. Zimmermann H, Freing A, Kaufhold F, Gaede G, Bohn E, Bock M, et al. Optic neuritis interferes with optical coherence tomography and magnetic resonance imaging correlations. Mult Scler. 2013;19:443-50.

34. Klistorner A, Graham EC, Yiannikas C, Barnett M, Parratt J, Garrick R, et al. Progression of retinal ganglion cell loss in multiple sclerosis is associated with new lesions in the optic radiations. Eur J Neurol. 2017;24:1392-8.

35. Sriram P, Wang C, Yiannikas C, Garrick R, Barnett M, Parratt J, et al. Relationship between optical coherence tomography and electrophysiology of the visual pathway in non-optic neuritis eyes of multiple sclerosis patients. PLoS One. 2014;9:e102546.

36. Behbehani R, Ahmed S, Al-Hashel J, Rousseff RT, Alroughani R. Sensitivity of visual evoked potentials and spectral domain optical coherence tomography in early relapsing remitting multiple sclerosis. Mult Scler Relat Disord. 2017;12:15-9.

37. Huang-Link YM, Fredrikson M, Link H. Benign multiple sclerosis is associated with reduced thinning of the retinal nerve fiber and ganglion cell layers in non-optic-neuritis eyes. J Clin Neurol. 2015;11:241-7.

38. Meltzer E, Sguigna PV, Subei A, Beh S, Kildebeck E, Conger D, et al. Retinal architecture and melanopsin-mediated pupillary response characteristics. JAMA Neurol. 2017;74:574.

39. Martinez-Lapiscina EH, Arnow S, Wilson JA, Saidha S, Preiningerova JL, Oberwahrenbrock T, et al. Retinal thickness measured with optical coherence tomography and risk of disability worsening in multiple sclerosis: a cohort study. Lancet Neurol. 2016;15:574-84.

40. Bsteh G, Hegen H, Teuchner B, Amprosi M, Berek K, Ladstätter $\mathrm{F}$, et al. Peripapillary retinal nerve fibre layer as measured by optical coherence tomography is a prognostic biomarker not only for physical but also for cognitive disability progression in multiple sclerosis. Mult Scler. 2017 Oct 1;1352458517740216.

41. Gabilondo I, Martínez-Lapiscina EH, Fraga-Pumar E, Ortiz-Perez $\mathrm{S}$, Torres-Torres R, Andorra M, et al. Dynamics of retinal injury after acute optic neuritis. Ann Neurol. 2015;77:517-28.

42. Kupersmith MJ, Garvin MK, Wang J-K, Durbin M, Kardon R. Retinal ganglion cell layer thinning within one month of presentation for optic neuritis. Mult Scler. 2015;22:641-8.

43. Lanzillo R, Cennamo G, Criscuolo C, Carotenuto A, Velotti N, Sparnelli $\mathrm{F}$, et al. Optical coherence tomography angiography retinal vascular network assessment in multiple sclerosis. Mult Scler J. 2017 Sep 21;135245851772946.

44. Spain RI, Liu L, Zhang X, Jia Y, Tan O, Bourdette D, et al. Optical coherence tomography angiography enhances the detection of optic nerve damage in multiple sclerosis. Br J Ophthalmol. 2017 Aug 16;bjophthalmol-2017-310477. 